\title{
openheart Impact of periodontitis as representative of chronic inflammation on long-term clinical outcomes in patients with atrial fibrillation
}

\author{
Sung II Im, ${ }^{1}$ Jinho Heo, ${ }^{2}$ Bong Joon Kim, ${ }^{1}$ Kyoung-Im Cho, ${ }^{1}$ Hyun Su Kim, ${ }^{1}$ \\ Jung $\mathrm{Ho} \mathrm{Heo},{ }^{1}$ Jin Yong Hwang ${ }^{3}$
}

To cite: Im SI, Heo J, Kim BJ, et al. Impact of periodontitis as representative of chronic inflammation on long-term clinical outcomes in patients with atrial fibrillation. Open Heart 2018;5: 000708 . doi:10.1136/

openhrt-2017-000708

SIl and JH contributed equally.

The abstract of this paper was presented in the 9th Asia Pacific Heart Rhythm Society scientific session as poster.

Received 30 August 2017 Revised 12 February 2018 Accepted 3 April 2018

\section{Check for updates}

${ }^{1}$ Division of Cardiology, Department of Internal Medicine, Kosin University Gospel Hospital Kosin University College of Medicine, Busan, Republic of Korea

${ }^{2}$ Department of Dentistry/Oral \& Maxillofacial Surgery, Kosin University Gospel Hospital, Kosin University College of Medicine, Busan, Republic of Korea ${ }^{3}$ Division of Cardiology, Department of Internal Medicine, Gyeongsang National University Hospital, Gyeongsang National University College of Medicine Jinju, Republic of Korea

Correspondence to Dr. Jung Ho Heo, Divisionof cardiology, Department of Internal Medicine, Kosin University GospelHospital, Kosin University College of Medicine, Busan 602-702, Republic ofKorea. ; duggymdc@gmail. com

\section{ABSTRACT}

Objectives Relationship between atrial fibrillation (AF) and inflammation was shown in previous studies. However, there was limited data about the association between the periodontitis and $A F$ in the long-term follow-up. The aim of this study was to evaluate the impact of periodontitis on long-term clinical outcomes in patients with AF. Methods The Kosin University echocardiography, ECG and periodontitis database were reviewed from 2013 to 2015 to identify patients with AF. Those patients were divided into two groups according to the presence of periodontitis and clinical events including any arrhythmic attack, thromboembolic and bleeding and death were collected during a median of 18 months.

Results Among 227 patients with AF, 47 (20.7\%) patients had periodontitis. Major adverse cardiac events (MACE) were significantly higher in patients with periodontitis compared with those without periodontitis $(p<0.001)$. Arrhythmias including AF, atrial tachycardia, atrial premature beat, ventricular tachycardia and ventricular premature beat also occurred in 44 (93.6\%) patients, which was higher significantly higher incidence in patients with periodontitis than in those without periodontitis $(p<0.001)$. In univariate analysis, age, $\mathrm{CHA}_{2} \mathrm{DS}_{2}-\mathrm{VASc}$, left atrial volume index (LAVi) and periodontitis were significantly associated with arrhythmic events and MACE including bleeding events, thromboembolic events, arrhythmic events and mortality. In multivariate analysis, LAVi $(p=0.005)$ and periodontitis $(p<0.001)$ were independent risk factors for arrhythmic events and periodontitis $(p<0.001)$ for MACE at the long-term followup.

Conclusions The periodontitis as representative of chronic inflammation was an independent predictor of arrhythmic events and MACE in patients with AF.

\section{INTRODUCTION}

Atrial fibrillation (AF) is the most common clinically significant arrhythmia in clinical practice. $\mathrm{AF}$ is associated with increased morbidity and mortality that primarily occur as a result of complications, such as thromboembolic events and heart failure. ${ }^{1}$

$\mathrm{AF}$ is often associated with other cardiovascular diseases (CVDs), including diabetes

\section{Key questions}

What is already known about this subject?

- In recent studies, there have been increasing evidence linking local and systemic inflammation to broad spectrum of cardiovascular condition, such as coronary artery disease and atrial fibrillation (AF). In addition, emerging data supported the association between inflammation and $\mathrm{AF}$.

What does this study add?

- AF patients with periodontitis, as representative of chronic inflammation, were more likely to develop arrhythmic events including AF, atrial tachycardia and atrial flutter AF and total any events than those without periodontitis.

How might this impact on clinical practice?

- The control of oral disease may improve inflammation and may be used as a possible therapeutic target to prevent embolic events and recurrence of arrhythmia in patients with AF.

mellitus, hypertension, congestive heart failure, ischaemic heart disease, valvular heart diseases and other cardiomyopathies. However, in $10 \%-15 \%$ of the cases, AF occurs in the absence of any such comorbidities and is determined as lone $\mathrm{AF}^{2{ }^{2}}$ In recent studies, there has been increasing evidence linking local and systemic inflammation to broad spectrum of cardiovascular condition, such as coronary artery disease and AF. In addition, emerging data supported the association between inflammation and $\mathrm{AF}^{45}$

Various dental procedures, routine oral hygiene efforts and even gentle mastication may lead to the entry of bacteria or bacterial endotoxins from the oral cavity into the bloodstream; therefore, bacterial infection, antigens, endotoxins and pro-inflammatory cytokines triggering a systemic response may represent the link between periodontitis and coronary artery disease. ${ }^{6}$ Adult periodontitis 
is a chronic inflammatory disease resulting from infection by specific microbial species. Local inflammation in oral cavity causes an enhanced inflammatory response at distant sites.

Recently, the relations between periodontitis and CVDs have been recognised. Periodontitis plays a role in the pathogenesis of atherosclerosis, and relationship between $\mathrm{AF}$ and inflammation has been shown in previous studies. $^{7}$

However, there have been limited data about the association between the periodontitis and $\mathrm{AF}$ in the longterm follow-up. The aim of this study was to evaluate the impact of periodontitis on long-term clinical outcomes in patients with AF.

\section{METHODS}

\section{Study populations}

We retrospectively reviewed the medical records of 428 patients with non-valvular AF at Kosin University Gospel Hospital from January 2013 to November 2015.

We included non-valvular $\mathrm{AF}$ patients with/without periodontitis. Patients with a history of AF documented by a standard ECG or Holter-ECG were enrolled.

Patients with a recent history of acute infection or inflammatory disease except periodontitis were excluded. Exclusion criteria were as follows: age $>80$ years; a history of cardiomyopathy, or valvular or congenital heart disease; hepatic or renal disease (known chronic liver disease or aspartate aminotransferase [AST] $>3$ times than normal range, more advanced chronic kidney disease [CKD] or stage 3); an acute cardiovascular or cerebrovascular event within the preceding three months; any major trauma or surgery within the preceding three months; hyperthyroidism; uncontrolled hypertension; malignancy; connective tissue disease or ischaemic heart disease.

Finally, 227 consecutive patients with non-valvular AF (mean age $59.6 \pm 10.9$ years, $68.7 \%$ male) were enrolled. And all patients were reviewed to evaluate major bleeding, thromboembolic events, arrhythmic events and death during follow-up according to the periodontitis. ${ }^{8}$ Trained and calibrated dentists examined the periodontal status of the patients. The WHO community periodontal index (CPI) was used to evaluate periodontitis and periodontitis was diagnosed when a CPI was greater than or equal to code $3{ }^{9}$

\section{Data collection}

After ECG and chest X-ray, cardiovascular status was evaluated for each patient using echocardiography, 24-hour Holter recordings and blood laboratory data from the initial visit, as determined by the attending physicians. From the database, the following information was collected: (1) patient data, including sex, age, height and weight; (2) cardiovascular risk factors, including hypertension and diabetes mellitus; (3) CVD status, including structural heart disease, congestive heart failure or a history of a disabling cerebral infarction or transient ischaemic attack (TIA); and (4) use of medication. We categorised the alcohol drinker was defined with moderate alcohol consumption (1-7 drinks/week).

\section{Definitions of AF and atrial arrhythmia}

In the present study, paroxysmal AF (PAF) at the initial visit was defined as sinus rhythm on ECG and previous diagnosis of PAF by referring physicians. Patients whose $\mathrm{AF}$ was estimated to continue for $\geq 7$ days after the initial visit were considered to have persistent $\mathrm{AF}(\mathrm{PeAF})$ originally and were excluded from the analysis. Chronic AF was defined as an ongoing long-term episode. Asymptomatic $\mathrm{AF}$ was defined as AF documented on 12-lead ECG during a visit, in the absence of any new symptoms such as palpitations, tachycardia, fatigue, malaise, shortness of breath on exertion, dyspnoea, chest pain, syncope or pre-syncope related to AF or other illnesses. During the follow-up period, the onset of AF was defined as the first time in which all ECGs indicated AF after $\geq 3$ consecutive ECGs at intervals of $\geq 1$ week after the initial examination. ${ }^{10}$ When an ECG could not be obtained thrice during the period, the physicians made a clinical judgement regarding the onset time of AF progression. We calculated the CHADS, score (congestive heart failure, hypertension, age $(\geq 75$ years), diabetes mellitus; one point each, and history of stroke or TIA; two points). The $\mathrm{CHA}_{2} \mathrm{DS}_{2}$-VASc score was also determined, which also includes vascular disease (previous myocardial infarction, complex aortic plaque and peripheral artery disease), age $65-74 .{ }^{11}$ HAS-BLED score was calculated (hypertension, abnormal renal/ liver function, stroke, bleeding history or predisposition, labile International Normalized Ratio [INR], elderly (>65), drugs/alcohol concomitantly—one point each). Atrial arrhythmia during follow-up was defined as atrial premature complex, atrial tachycardia and atrial flutter.

\section{Clinical endpoints}

The primary clinical endpoint was major adverse cardiac events (MACE) including major bleeding defined as that which was clinically overt and associated with any of the following: fatal outcome; involvement of a critical anatomic site (intracranial, spinal, ocular, pericardial, articular, retroperitoneal or intramuscular with compartment syndrome); fall in haemoglobin concentration $>2 \mathrm{~g} / \mathrm{dL}$; transfusion of $>2 \mathrm{U}$ of whole blood; or packed red blood cells, thromboembolic events, which was the composite of stroke, TIA, systemic emboli and death during follow-up. And the secondary endpoint was arrhythmic events including AF, atrial tachycardia, atrial premature beat, ventricular tachycardia and ventricular premature beat.

\section{Transthoracic echocardiography}

All enrolled subjects underwent two-dimensional transthoracic echocardiography (TTE). All examinations were performed using a commercially available Vivid V.9 (GE Medical System, Vingmed, Horten, Norway) ultrasound system. All recorded echocardiograms were 
measured and interpreted with clinical information blinded using a computerised offline analysis station (Echopac V.6.3.4; GE Medical System).

\section{Statistical analysis}

For continuous data, statistical differences were evaluated using Student's t-test or the Mann-Whitney U test, depending on the data distribution. Categorical variables are presented as frequencies (per cent) and were analysed using the $\chi^{2}$ test. To determine whether any of the variables were independently related to periodontitis, a multivariate analysis of variables with a $\mathrm{p}$ value $<0.05$ in the univariate analysis was performed using linear logistic regression analysis. All statistical analyses were conducted using SPSS statistical software, V.19.0, and statistical significance was set at $\mathrm{p}<0.05$ (two-sided).

\section{RESULTS}

The baseline demographics for both groups are listed in table 1. This study consisted of $47 \mathrm{AF}$ subjects with periodontitis and $180 \mathrm{AF}$ subjects without periodontitis. Baseline characteristics were not statistically different between the periodontitis subjects and the non-periodontitis subjects, except for age $(\mathrm{p}<0.001)$, HAS-BLED scores $(p<0.001)$ and $\operatorname{PeAF}(p=0.043)$. There was no patient with chronic AF in both groups-total 70 patients (30.8\%) with PAF and 157 patients (69.2\%) with PeAF.

For the history of medications, there was no difference between the periodontitis subjects and the non-periodontitis subjects, except for aspirin $(\mathrm{p}<0.001)$ and vitamin $\mathrm{K}$ antagonist $(\mathrm{VKA}, \mathrm{p}<0.001)$, that were used more often in the non-periodontitis group than in the periodontitis group.

In baseline laboratory findings, white blood cell counts (WBC, p<0.001), neutrophil/lymphocyte ratio (NLR, $\mathrm{p}=0.001$ ) and high-sensitive C-reactive protein (hsCRP, $\mathrm{p}=0.048$ ) levels were higher in the periodontitis group. And there was no difference of the echocardiographic findings between the periodontitis subjects and the non-periodontitis subjects, except for left atrial volume index (LAVi), E, A, E', E/A and E/E' velocity at baseline (table 1).

All patients were reviewed to evaluate arrhythmic events including AF, atrial tachycardia, atrial premature beat, ventricular tachycardia and ventricular premature beat, and MACE including major bleeding, thromboembolic events including stroke, TIA, systemic emboli and death during follow-up.

The clinical outcomes in patients with $\mathrm{AF}$ at 18-month follow-up are shown in table 2. MACE (40 patients $(85.1 \%), \mathrm{p}<0.001)$, total thromboembolic events (14 patients $(29.8 \%), \mathrm{p}<0.001)$ and arrhythmic events (44 patients $(93.6 \%), \mathrm{p}<0.001)$ were higher in patients with periodontitis compared with those without periodontitis during a mean follow-up of $18.1 \pm 10.2$ months.

Total oral bacterial culture-positive (\%) are 20 patients $(42.6 \%)$ with periodontitis. a-Streptococci $(70 \%)$ are the most common oral pathogen for periodontitis. Oral fungal culture-positive (\%) (Candida albicans $(75 \%)$ ) are four patients in table 3 .

In univariate analysis, periodontitis, $\mathrm{CHA}_{2} \mathrm{DS}_{2}$-VASc score, HAS-BLED score, LAVi and $\mathrm{E} / \mathrm{E}^{\prime}$ velocity were significantly associated with MACE including bleeding events, thromboembolic events, arrhythmic events and mortality. In multivariate analysis, periodontitis $(p<0.001)$ was independent risk factors for MACE in table $4 \mathrm{~A}$ at the long-term follow-up.

In univariate analysis, periodontitis, $\mathrm{CHA}_{2} \mathrm{DS}_{2}$-VASc score, HAS-BLED score, LAVi, E' velocity, WBC counts and NLR were significantly associated with arrhythmic events including AF, atrial flutter, atrial tachycardia, ventricular premature beat, ventricular tachycardia and sick sinus syndrome. In multivariate analysis, periodontitis $(\mathrm{p}=0.030)$ and LAVi $(p=0.019)$ were independent risk factors for arrhythmic events in table $4 \mathrm{~B}$ at the long-term follow-up.

Flow diagram of our study is shown in figure 1. KaplanMeier curves show that the event-free survival from MACE including bleeding events, thromboembolic events, arrhythmic events and mortality $(\mathrm{p}<0.001$; figure $2 \mathrm{~A})$ event-free survival from arrhythmic events $(p<0.001$; figure 2B) are lower in patients with periodontitis compared with those without periodontitis at 18-month follow-up.

\section{DISCUSSION}

In this study, we showed that the periodontitis in patients with $\mathrm{AF}$ is associated with higher incidence of arrhythmic events and MACE including major bleeding, thromboembolic events including stroke, TIA, systemic emboli and death in the long-term follow-up, suggesting more intensive medical therapy to control periodontitis as representative of chronic inflammation with close clinical follow-up will be required.

The link between periodontal disease and CVD is complex and many publications have provided support to the hypothesis of a causal association. ${ }^{12}$ Periodontitis induces a peripheral inflammatory and immune response, reflected in elevated CRP and IgA class antibodies to periodontal pathogens. The prevalence of CVD seems to be the highest in those individuals in whom periodontitis coexists with elevated CRP levels. And elevated inflammation cytokines contribute to the pathogenesis of AF. The CRP levels were associated with the burden of AF. This may be due to genetic reasons and may also apply to chronic low-grade infections. ${ }^{7}$ And several studies have shown that there are many studies that have indicated that inflammation might play a significant role in the initiation, maintenance and perpetuation of $\mathrm{AF}^{13}$ In our study, there were higher WBC, NLR and hsCRP levels in the periodontitis group compared with those in the non-periodontitis group, which is consistent with a previous study. ${ }^{14}$ 
Table 1 Baseline clinical characteristics in patients with atrial fibrillation (AF) according to the presence of periodontitis

\begin{tabular}{|c|c|c|c|}
\hline Variables & $\begin{array}{l}\text { Periodontitis group } \\
(n=47)\end{array}$ & $\begin{array}{l}\text { Non-periodontitis group } \\
(n=180)\end{array}$ & $P$ values \\
\hline Age (years) & $71.1 \pm 8.7$ & $56.7 \pm 9.4$ & $<0.001$ \\
\hline Gender (male, \%) & 27 (61.4) & 129 (72.5) & 0.197 \\
\hline $\operatorname{PeAF}(\%)$ & $11(25.0)$ & $59(32.3)$ & 0.043 \\
\hline DM (\%) & $12(27.3)$ & $26(14.5)$ & 0.071 \\
\hline HTN (\%) & $28(65.1)$ & $98(54.7)$ & 0.235 \\
\hline CHF (\%) & $5(10.6)$ & $19(10.6)$ & 1.000 \\
\hline CVA (\%) & $3(1.7)$ & $3(6.8)$ & 0.093 \\
\hline $\mathrm{CHADS}_{2}$ & $1.3 \pm 0.5$ & $1.4 \pm 0.4$ & 0.789 \\
\hline $\mathrm{CHA}_{2} \mathrm{DS}_{2}$-VASC & $1.8 \pm 1.5$ & $1.8 \pm 0.7$ & 0.845 \\
\hline HAS-BLED score & $2.9 \pm 1.6$ & $1.5 \pm 0.5$ & $<0.001$ \\
\hline COPD (\%) & $1(2.3)$ & $0(0)$ & 0.197 \\
\hline Alcohol (\%) & $6(14.0)$ & $48(26.8)$ & 0.112 \\
\hline Smoking (\%) & $5(11.6)$ & $30(16.8)$ & 0.491 \\
\hline \multicolumn{4}{|l|}{ Medication } \\
\hline \multicolumn{4}{|l|}{ Antiarrhythmics (\%) } \\
\hline Amiodarone (\%) & $2(4.0)$ & $6(3.5)$ & 1.000 \\
\hline Propafenone (\%) & $6(12.7)$ & $25(14.0)$ & 0.450 \\
\hline Flecainide (\%) & $7(1.5)$ & $27(1.5)$ & 1.000 \\
\hline Digoxin (\%) & $2(4.3)$ & $11(6.1)$ & 1.000 \\
\hline Beta-blocker (\%) & $11(23.2)$ & $43(24.0)$ & 0.419 \\
\hline CCB $(\%)$ & $13(28.8)$ & $44(24.5)$ & 0.365 \\
\hline ARB and ACEi (\%) & $12(25.5)$ & $42(23.5)$ & 0.407 \\
\hline Statins (\%) & $0(0)$ & $0(0)$ & 1.000 \\
\hline Aspirin (\%) & $4(8.5)$ & $64(35.6)$ & $<0.001$ \\
\hline VKA & $12(25.5)$ & $101(56.1)$ & $<0.001$ \\
\hline \multicolumn{4}{|l|}{ Laboratory findings } \\
\hline WBC $\left(10^{3} / \mu \mathrm{L}\right)$ & $8.7 \pm 3.5$ & $6.6 \pm 2.8$ & $<0.001$ \\
\hline NLR & $7.8 \pm 6.5$ & $2.8 \pm 2.2$ & 0.001 \\
\hline Creatinine (mg/dL) & $1.1 \pm 1.0$ & $0.9 \pm 0.7$ & 0.113 \\
\hline hsCRP (mg/dL) & $1.6 \pm 0.3$ & $1.1 \pm 0.4$ & 0.048 \\
\hline \multicolumn{4}{|l|}{ Echo parameters } \\
\hline LVEF (\%) & $62.0 \pm 9.7$ & $63.8 \pm 8.3$ & 0.203 \\
\hline LVIDs (mm) & $30.6 \pm 5.9$ & $32.0 \pm 5.6$ & 0.151 \\
\hline LVIDd (mm) & $45.6 \pm 9.7$ & $46.3 \pm 5.9$ & 0.245 \\
\hline IVSD (mm) & $11.7 \pm 2.8$ & $11.2 \pm 1.8$ & 0.127 \\
\hline LVPWD (mm) & $10.4 \pm 2.3$ & $10.2 \pm 1.5$ & 0.441 \\
\hline LAVi $\left(\mathrm{mL} / \mathrm{m}^{2}\right)$ & $44.2 \pm 8.8$ & $36.0 \pm 13.1$ & $<0.001$ \\
\hline E velocity $(\mathrm{cm} / \mathrm{s})$ & $0.9 \pm 0.4$ & $0.8 \pm 0.2$ & 0.004 \\
\hline A velocity $(\mathrm{cm} / \mathrm{s})$ & $0.8 \pm 0.2$ & $0.6 \pm 0.2$ & $<0.001$ \\
\hline$E / A$ & $1.0 \pm 0.7$ & $1.4 \pm 0.7$ & 0.016 \\
\hline$E^{\prime}$ & $0.05 \pm 0.2$ & $0.1 \pm 0.1$ & 0.001 \\
\hline$A^{\prime}$ & $0.1 \pm 0.02$ & $0.1 \pm 0.02$ & 0.090 \\
\hline$E / E^{\prime}$ & $13.8 \pm 6.7$ & $9.8 \pm 4.8$ & $<0.001$ \\
\hline
\end{tabular}

Values are mean \pm SD (range).

A, peak velocity of the late filling wave due to atrial contraction; A', late diastolic mitral annulus velocity; ARB, angiotensin II receptor blocker; CCB, calcium channel blocker; CHF, congestive heart failure; COPD, chronic obstructive pulmonary disease; CRF, chronic renal failure; CVA, cerebrovascular accident; DM, diabetes mellitus; $\mathrm{E}$, the peak mitral flow velocity of the early rapid filling wave; E', early diastolic mitral annulus velocity; HTN, hypertension; hsCRP, high-sensitive C-reactive protein; IVSd, interventricular septal diameter; LAVi, left atrial volume index; LVEF, left ventricular ejection fraction; LVIDs, left ventricular systolic diameter; LVIDd, left ventricular diastolic diameter; NLR, neutrophil/lymphocyte ratio; PeAF, persistent atrial fibrillation; VKA, vitamin K antagonist; WBC, white blood cell count. 
Table 2 Clinical outcomes in patients with atrial fibrillation (AF) according to the periodontitis at 18-month follow-up

\begin{tabular}{|c|c|c|c|}
\hline Variables & $\begin{array}{l}\text { Periodontitis group } \\
(n=47)\end{array}$ & $\begin{array}{l}\text { Non-periodontitis group } \\
(n=180)\end{array}$ & $P$ values \\
\hline Follow-up duration (months) & $16.9 \pm 10.3$ & $19.1 \pm 15.0$ & 0.349 \\
\hline MACE (\%) & $40(85.1)$ & $44(24.6)$ & $<0.001$ \\
\hline Total death (\%) & $1(2.3)$ & $1(0.6)$ & 0.356 \\
\hline Cardiac death (\%) & $1(2.3)$ & $1(0.6)$ & \\
\hline Total thromboembolic events (\%) & $14(29.8)$ & $1(0.6)$ & $<0.001$ \\
\hline CVA (new onset, \%) & $12(25.8)$ & $0(0)$ & \\
\hline Peripheral thromboembolism (\%) & $2(4.0)$ & $1(0.6)$ & \\
\hline Bleeding complications (\%) & $3(6.8)$ & $13(7.3)$ & 1.000 \\
\hline Arrhythmic events (\%) & $44(93.6)$ & $31(17.4)$ & $<0.001$ \\
\hline SVT including AF, AFL or ATach (\%) & $44(93.6)$ & $30(16.9)$ & \\
\hline VPC or VTach (\%) & $0(0)$ & $1(0.5)$ & \\
\hline \multicolumn{4}{|l|}{ Treatment of AF } \\
\hline DC cardioversion for rhythm control (\%) & $7(14.5)$ & $18(10.5)$ & 0.042 \\
\hline
\end{tabular}

Values are mean $\pm \mathrm{SD}$ (range).

AFL, atrial flutter; ATach, atrial tachycardia; CVA, cerebrovascular accidents; MACE, major adverse cardiac events; SVT, supraventricular tachycardia; VPC, ventricular premature complex; VTach, ventricular tachycardia.

Chronic inflammation and autonomic imbalance have been proposed as plausible pathophysiological mechanisms of AF. ${ }^{15}$ And structural and electrical alternations have been reported in the development and perpetuation of $\mathrm{AF}$ and local inflammation resulting from periodontitis, including inflammatory infiltration cardiomyocytes hypertrophy and disturbed atrial structure. ${ }^{7}$

Periodontal bacteria and bacterial components have easy access to the periodontal connective tissue and enter the blood circulation frequently. Previous study reported that bacteria such as Porphyromonas gingivalis and Prevotella intermedia stimulate a humoural response and antibodies generated in response to the bacterium may indeed contribute to tissue damage. ${ }^{16}$ Once the damage is confirmed in the myocardium, it may be responsible for the substrate of arrhythmia. In our study,

Table 3 Periodontal pathogens in atrial fibrillation patients with periodontitis

\begin{tabular}{ll}
\hline Variables & $\begin{array}{l}\text { Periodontitis } \\
\text { group }(\mathbf{n}=47)\end{array}$ \\
\hline Total oral bacterial culture-positive (\%) & $20(42.6)$ \\
\hline Periodontal pathogens (\%) & \\
a-Streptococci (\%) & $14(70.0)$ \\
\hline Klebsiella pneumoniae (\%) & $2(10.0)$ \\
\hline Escherichia coli (\%) & $2(10.0)$ \\
\hline Staphylococcus aureus (\%) & $1(5.0)$ \\
\hline Staphylococcus salivarius (\%) & $1(5.0)$ \\
\hline Oral fungal culture-positive (\%) & $4(8.5)$ \\
Candida albicans (\%) & $3(75.0)$ \\
Candida tropicalis (\%) & $1(25.0)$ \\
\hline
\end{tabular}

total oral bacterial culture-positive $(\%)$ are 20 patients $(42.6 \%)$ with periodontitis. a-Streptococci $(70 \%)$ are the most common oral pathogen for periodontitis. Oral fungal culture-positive (\%) (C. albicans $(75 \%)$ ) are four patients. And there was no difference of clinical outcomes among the periodontal pathogens in patients with periodontitis in our study. Unfortunately, there are no data currently on ways to risk-stratify these patients according to periodontal pathogens in this study because of small number of patients.

In our study, we hypothesised that periodontitis may also be a risk factor for $\mathrm{AF}$ on the basis of the following facts. First of all, periodontitis may impair endothelial function and periodontitis can be associated with oxidative stress both locally and peripherally. Endothelial dysfunction and oxidative stress are both involved in the pathophysiology of AF. CRP downregulates endothelial nitric oxide (NO) synthase transcription in endothelial cells and destabilises endothelial NO synthase messenger RNA, resulting in decreased NO release. ${ }^{17}$ Previous study reported that many proofs have implied that AF may be an inflammatory disease. Inflammation caused by sterile pericarditis increased the CRP, decreased atrial effective refractory periods, delayed atrial conduction time and $\mathrm{AF}$ duration. 18

Based on the predictors of bleeding, the HAS-BLED score was developed using a 'real-world' cohort of 3978 European subjects with AF from the EuroHeart Survey. ${ }^{19}$ In our study, there was also trend of higher HAS-BLED score in the periodontitis group compared with those in the non-periodontitis group. However, there was no difference of the incidences of bleeding events $(\mathrm{p}=0.239)$ including minor bleeding-gingival bleeding according to periodontitis at 18-month clinical follow-up 
Table 4 Univariate and multivariate Cox analyses for major adverse cardiac events (A) and arrhythmic events (B) in patients with atrial fibrillation according to the periodontitis at 18-month follow-up.

\begin{tabular}{|c|c|c|c|c|}
\hline \multirow{2}{*}{$\begin{array}{l}\text { Variable } \\
\text { N (\%) }\end{array}$} & \multicolumn{2}{|l|}{ Univariate analysis } & \multicolumn{2}{|l|}{ Multivariate analysis } \\
\hline & OR $(95 \% \mathrm{Cl})$ & $P$ values & OR $(95 \% \mathrm{Cl})$ & $P$ values \\
\hline \multicolumn{5}{|c|}{ (A) Major adverse cardiac events } \\
\hline Periodontitis & 17.532 (7.330 to 41.937$)$ & $<0.001$ & 17.776 (3.459 to 91.344$)$ & 0.001 \\
\hline $\mathrm{CHA}_{2} \mathrm{DS}_{2}-\mathrm{VASC}$ & 1.280 (1.010 to 1.622$)$ & 0.041 & & \\
\hline HAS-BLED & 2.119 (1.590 to 2.825$)$ & $<0.001$ & & \\
\hline LAVi & 1.030 (1.007 to 1.054$)$ & 0.012 & & \\
\hline $\mathrm{E} / \mathrm{E}^{\prime}$ & 1.060 (1.060 to 1.118$)$ & 0.029 & & \\
\hline WBC counts & $1.000(0.998$ to 1.000$)$ & 0.588 & & \\
\hline NLR & 1.014 (0.980 to 1.051$)$ & 0.420 & & \\
\hline hsCRP & 0.065 (0.001 to 7.037$)$ & 0.253 & & \\
\hline \multicolumn{5}{|c|}{ (B) Arrhythmic events } \\
\hline Periodontitis & 40.688 (11.780 to 140.533$)$ & $<0.001$ & 9.185 (1.241 to 67.964$)$ & 0.030 \\
\hline $\mathrm{CHA}_{2} \mathrm{DS}_{2}-\mathrm{VASC}$ & 1.434 (1.089 to 1.888$)$ & 0.010 & & \\
\hline HAS-BLED & 3.608 (2.242 to 5.805$)$ & $<0.001$ & & \\
\hline LAVi & 1.107 (1.064 to 1.151$)$ & $<0.001$ & 1.055 (1.009 to 1.103$)$ & 0.019 \\
\hline $\mathrm{E}^{\prime}$ & $1.113(1.041$ to 1.191$)$ & 0.002 & & \\
\hline WBC count & 1.000 (1.000 to 1.001$)$ & 0.011 & & \\
\hline NLR & 1.102 (1.003 to 1.169$)$ & 0.006 & & \\
\hline
\end{tabular}

$\mathrm{E}$, the peak mitral flow velocity of the early rapid filling wave; E', early diastolic mitral annulus velocity; hsCRP, high-sensitive C-reactive protein; LAVi, left atrial volume index; NLR, neutrophil/lymphocyte ratio; WBC, white blood cell count.

in Kaplan-Meier analysis. And there was lower incidence of VKA medication in the periodontitis group $(\mathrm{p}<0.001)$, which reflect that the dentists did not want to use the anticoagulation medication during the treatment of periodontitis.

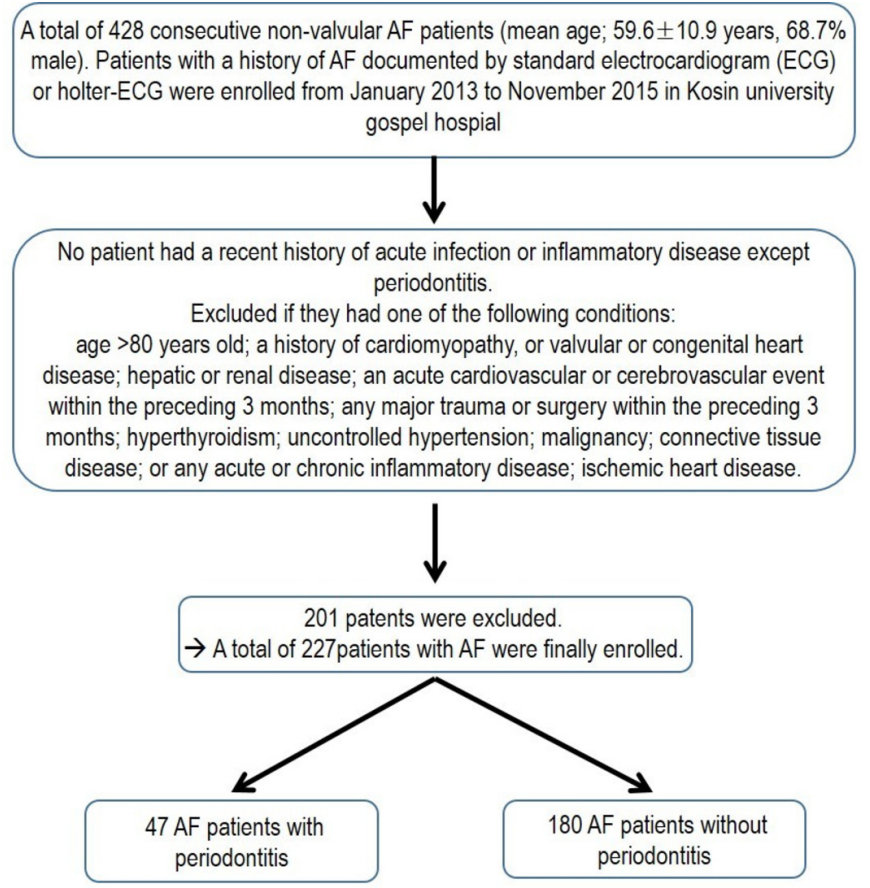

Figure 1 Flow diagram. AF, atrial fibrillation.
It is of interest that although there was no difference of the incidences of hospitalisation rate $(\mathrm{p}=0.721)$, total death $(\mathrm{p}=0.356)$ and bleeding complication $(p=1.000)$ in both groups, the incidences of MACE $(p<0.001)$, total thromboembolic events $(\mathrm{p}<0.001)$, arrhythmic events $(\mathrm{p}<0.001)$ and DC cardioversion for rhythm control ( $p=0.042)$ were higher in the periodontitis group compared with the non-periodontitis group.

In our study, various factors were associated with arrhythmic events in patients with $\mathrm{AF}$, including periodontitis, $\mathrm{CHA}_{2} \mathrm{DS}_{2}-\mathrm{VASc}$ score, HAS-BLED score, LAVi and $\mathrm{E}^{\prime}$ velocity on TTE. In multivariate analysis, periodontitis and LAVi were independent predictors for arrhythmic events in patients with $\mathrm{AF}$ at 18-month clinical follow-up.

Previous reports that demonstrated that PeAF became more likely, which led to the adoption of the now oft-quoted adage 'AF begets $\mathrm{AF}^{2}{ }^{20}$ In our study, there were higher incidences of PeAF $(p=0.043)$ in patients with non-periodontitis. However, at 18-month follow-up, arrhythmic events $(\mathrm{p}<0.001)$ including AF, atrial tachycardia and atrial flutter were also higher in the periodontitis group, which is consistent with a previous study ${ }^{21}$ that both oxidants and inflammation play an important role in the initiation and maintenance of AF.

This is the first study to evaluate the impact of periodontitis on long-term prognosis in patients with $\mathrm{AF}$ and validate a risk stratification to assess the probability of arrhythmic events. In our study, the periodontitis in patients with $\mathrm{AF}$ is associated with higher incidence of 

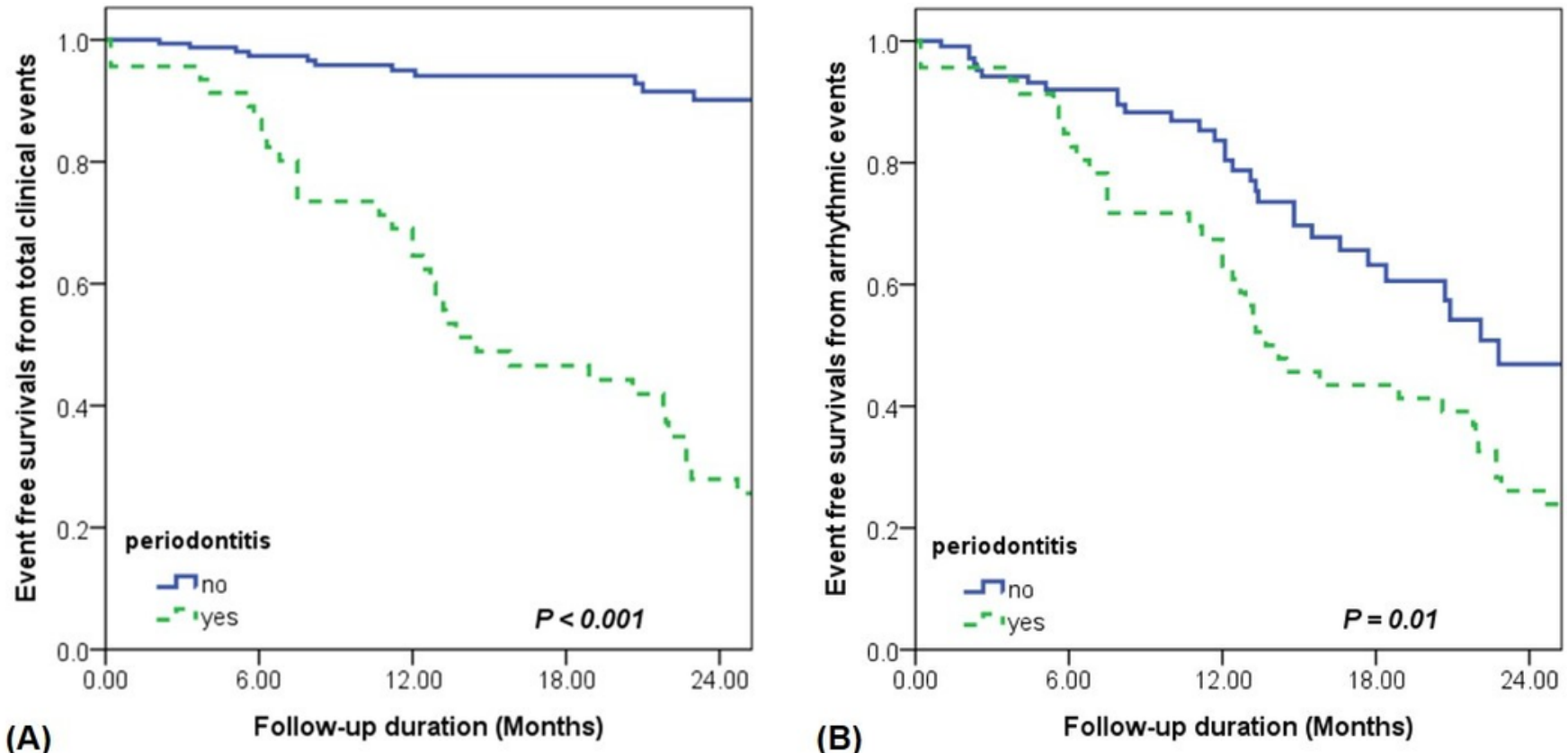

Periodontitis (n)

$\begin{array}{ll}\text { No } & 180 \\ \text { Yes } & 47\end{array}$

80
39

51

25

Periodontitis (n)

Figure 2 Kaplan-Meier analysis for event-free survival in patients with atrial fibrillation according to the presence of periodontitis: (A) major adverse cardiac events including bleeding events, thromboembolic events, arrhythmic events and mortality; (B) arrhythmic events.

MACE and arrhythmic events in the long-term follow-up, suggesting that, although the mechanism remains unclear, the control of oral disease may improve inflammation and may be used as a possible therapeutic target to prevent embolic events and recurrence of arrhythmia in patients with AF.

Further prospective studies are needed to determine if a true causal mechanism exists between periodontitis and $\mathrm{AF}$, as well as to access whether the mechanisms is dependent on a specific subtype of AF.

\section{Study limitations}

There are some limitations to our study. First, this was a single-centre, retrospective study derived from a realworld practice with inherent limitations. Small numbers of patients with periodontitis were included in this study. Because a half of total patients included at baseline had no medical record or history of dental procedure, therefore, we excluded those patients. Hence, the results of our study should be considered as hypothesis generating, and future prospective studies are warranted to confirm our results. Second, asymptomatic episodes of AF may not have been recognised because AF recurrence was based on clinical symptoms and ambulatory monitoring for a short period. Third, the investigators may not screen $\mathrm{AF}$ patients with asymptomatic periodontitis or treated for periodontitis in other clinics. Fourth, patients with periodontitis could not be treated continuously. Therefore, there was limitation to generate the direct correlation of periodontitis treatment with clinical outcomes. However, chronic inflammation and autonomic imbalance have been proposed as plausible pathophysiological mechanisms of AF. And this study has presented a new vision for the periodontitis focusing on the arrhythmogenic effects beyond local chronic inflammation.

\section{CONCLUSION}

The periodontitis as representative of chronic inflammation was an independent predictor of arrhythmic events and MACE in patients with AF in the long-term follow-up, suggesting that the control of oral disease may improve inflammation and may be used as a possible therapeutic target to prevent embolic events and recurrence of arrhythmia in patients with AF.

Acknowledgements The authors thank all the members of the Division of Cardiology, Department of Internal Medicine, Kosin University Gospel Hospital.

Contributors Conception and coordination of the study and data review: SII and JH. Design of ethical issues: BJK. Acquisition of data: SII and K-IC. Statistical analysis: SII and HSK. Manuscript preparation: SII, JHH and JYH. Manuscript approval: all authors.

Funding The authors have not declared a specific grant for this research from any funding agency in the public, commercial or not-for-profit sectors.

Competing interests None declared.

Patient consent Obtained.

Ethics approval The study protocol conforms to the ethical guidelines of the 1975 Declaration of Helsinki, and the research protocol was approved by the ethics committee of Kosin University Gospel Hospital.

Provenance and peer review Not commissioned; externally peer reviewed.

Data sharing statement № additional data are available.

Open Access This is an Open Access article distributed in accordance with the Creative Commons Attribution Non Commercial (CC BY-NC 4.0) license, which 
permits others to distribute, remix, adapt, build upon this work non-commercially, and license their derivative works on different terms, provided the original work is properly cited and the use is non-commercial. See: http://creativecommons.org/ licenses/by-nc/4.0/

(c) Article author(s) (or their employer(s) unless otherwise stated in the text of the article) 2018. All rights reserved. No commercial use is permitted unless otherwise expressly granted.

\section{REFERENCES}

1. Senoo K, Suzuki S, Otsuka T, et al. Progression to the persistent form in asymptomatic paroxysmal atrial fibrillation. Circ $J$ 2014;78:1121-6.

2. Lloyd-Jones DM, Wang TJ, Leip EP, et al. Lifetime risk for development of atrial fibrillation: the Framingham Heart Study. Circulation 2004;110:1042-6.

3. Im SI, Chun KJ, Park SJ, et al. Long-term Prognosis of Paroxysmal Atrial Fibrillation and Predictors for Progression to Persistnt or Chronic Atrial Fibrillation in the Korean Population. J Korean Med Sci 2015;30:895-902.

4. Gami AS, Hodge DO, Herges RM, et al. Obstructive sleep apnea, obesity, and the risk of incident atrial fibrillation. J Am Coll Cardiol 2007;49:565-71.

5. Lazzerini PE, Capecchi PL, Laghi-Pasini F. Systemic inflammation and arrhythmic risk: lessons from rheumatoid arthritis. Eur Heart $J$ 2017;38:ehw208.

6. Spahr A, Klein E, Khuseyinova N, et al. Periodontal infections and coronary heart disease: role of periodontal bacteria and importance of total pathogen burden in the Coronary Event and Periodontal Disease (CORODONT) study. Arch Intern Med 2006;166:554-9.

7. Yu G, Yu Y, Li YN, et al. Effect of periodontitis on susceptibility to atrial fibrillation in an animal model. $J$ Electrocardiol 2010;43:359-66.

8. Tran DT, Gay I, Du XL, et al. Assessment of partial-mouth periodontal examination protocols for periodontitis surveillance. J Clin Periodontol 2014;41:846-52.
9. Tran DT, Gay I, Du XL, et al. Assessing periodontitis in populations: a systematic review of the validity of partial-mouth examination protocols. J Clin Periodontol 2013;40:1064-71.

10. Pappone C, Radinovic A, Manguso F, et al. Atrial fibrillation progression and management: a 5-year prospective follow-up study. Heart Rhythm 2008:5:1501-7.

11. Lip GY, Nieuwlaat R, Pisters R, et al. Refining clinical risk stratification for predicting stroke and thromboembolism in atrial fibrillation using a novel risk factor-based approach: the euro heart survey on atrial fibrillation. Chest 2010;137:263-72.

12. Ueno M, Izumi $\mathrm{Y}$, Kawaguchi $\mathrm{Y}$, et al. Prediagnostic plasma antibody levels to periodontopathic bacteria and risk of coronary heart disease. Int Heart J 2012;53:209-14.

13. Xu Y, Sharma D, Li G, et al. Atrial remodeling: new pathophysiological mechanism of atrial fibrillation. Med Hypotheses 2013;80:53-6.

14. Lin $\mathrm{K}$, Chen $\mathrm{X}$, Zhang $\mathrm{L}$, et al. Proton pump inhibitors as also inhibitors of atrial fibrillation. Eur J Pharmacol 2013;718:435-40.

15. Velagapudi $P$, Turagam MK, Leal MA, et al. Atrial fibrillation and acid reflux disease. Clin Cardiol 2012;35:180-6.

16. de Boer SP, Cheng JM, Rangé $\mathrm{H}$, et al. Antibodies to periodontal pathogens are associated with coronary plaque remodeling but not with vulnerability or burden. Atherosclerosis 2014;237:84-91.

17. Verma S, Wang $\mathrm{CH}$, Li SH, et al. A self-fulfilling prophecy: C-reactive protein attenuates nitric oxide production and inhibits angiogenesis. Circulation 2002;106:913-9.

18. Liu T, Li G, Li L, et al. Association between C-reactive protein and recurrence of atrial fibrillation after successful electrical cardioversion: a meta-analysis. J Am Coll Cardiol 2007;49:1642-8.

19. Pisters $R$, Lane DA, Nieuwlaat $R$, et al. A novel user-friendly score (HAS-BLED) to assess 1-year risk of major bleeding in patients with atrial fibrillation: the Euro Heart Survey. Chest 2010;138:1093-100.

20. Lu Z, Scherlag BJ, Lin J, et al. Atrial fibrillation begets atrial fibrillation: autonomic mechanism for atrial electrical remodeling induced by short-term rapid atrial pacing. Circ Arrhythm Electrophysiol 2008;1:184-92.

21. Issac TT, Dokainish $\mathrm{H}$, Lakkis NM. Role of inflammation in initiation and perpetuation of atrial fibrillation: a systematic review of the published data. J Am Coll Cardiol 2007;50:2021-8. 